\title{
The researching of the airport intelligent information network construction and power control which based on 4G technology
}

\author{
Wangxun $^{1, a}$, Chenlei ${ }^{1, b}$, Duyingjie ${ }^{1, c}$, Caijiale $^{2, d}$ \\ ( 1 Chengdu aeronautic polytechnic,Chengdu Sichuan China 610100) \\ (2 The southwest branch of Air china Chengdu Sichuan China 610202)

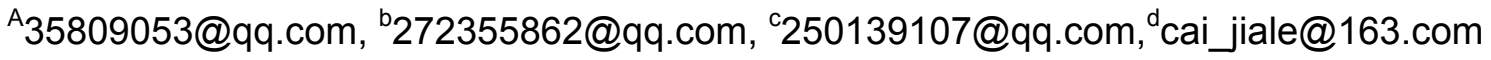

Keywords: fiber ring network; TD-LTE; positive and reverse power control

\begin{abstract}
According to the needs of intelligent and information management of modern airport , planning and implementation of the information platform of intelligent network system of Airport in southwest China. The backbone of this network structure using fiber ring network, wireless access network using TD-LTE devices. For avoid the interference to the presence radio communication equipment and commercial networks in airport. How to use positive and reverse power control technology of wireless access network for effective power management and ensure the airport communication smooth and security has been researched. Provide beneficial reference and theoretical support for the practice of large-scale comprehensive communication network system engineering in airport.
\end{abstract}

\section{Introduction}

Mobile Internet, indoor navigation, gridding , 4G technology, big data and cloud computing and other new technology are constantly emerging and they offer a variety of means and approaches to informationalized and intelligent management to airport. With highly informationalized airport, the passengers can use the information display system and intelligent mobile phone to get travel information more timely and shorten the waiting time. Using RFID Internet of Things technology, luggage transportation can realize real-time tracking and reduce the improper handling and lost of luggage. Using LBS (location-based services) and the e -beacon technology to get indoor navigation and various kinds of pushing and supporting service[1].

The data exchange of above-mentioned subsystems all need a mobile communication system platform with advanced and reliable technology, controlled cost, high safety and strong universality to be a support. To be equipped with the TD-LTE technology of our fully independent intellectual property rights not only can completely meet the above requirements, but also can access to commercial 4G network of China Mobile Communications Corporation seamlessly through the gateway. It is an ideal wireless access network system platform of establishing intelligent information network of airport.

Due to the complex electromagnetic environment of the airport, numerous all kinds of radio equipments, it is very likely to appear the case of cross interference. It requires the mobile communication system to have the ability of meeting the basic communication and reduce the transmission power as far as possible with effective power control ability. And the TD-LTE meets the demand with its advanced forward and reverse power control ability and it has become the solid choice of wireless access technology of the system .

\section{The overall planning and construction of airport intelligent information network}

Airport intelligent information network platform is to integrate original dispersed and independent subsystems(such as check-in, luggage transportation, boarding gate guide, indoor navigation, etc.), through the mobile Internet, cloud computing, optical network, RFID, Internet of things and other new technologies and new methods to realize the airport information management to be intelligentized, mobile, internet based and improve management level of the airport. 


\subsection{Construction of core backbone network}

Due to the large amount of information interaction between each subsystem in the platform, the peak exchange rate can reach TB level. Therefore, it is of high requirements to the reliability, real-time, high speed and security of the backbone network transmission. And as a new generation of communication medium, optical fiber is with the characteristics of low loss, broad bandwidth, high speed and strong anti-jam capability and it fully meets the requirements of being the core network media of the platform.

The platform is configured with FDDI (fiber distributed data interface) as a core topological structure and it adopts Gpon (Gigabit-Capable Passive Optical Network) as a core network for data transmission. The transmission rate can reach TB level and it has good transmission stability and security.

Subnet adopts EPON (Ethernet Passive Optical Network) to access core optical fiber ring network. It configure firewalls between subnets and backbones to defense DDOS attacks and the generation of network storm. $4 \mathrm{G}$ core network PS domain was also placed in the core optical fiber ring network and it uses the gateway to connect with China mobile commercial network. There configured firewalls between the core network and commercial network to defense network attacks.

\subsection{Wireless access network construction}

To achieve sub-network wireless access to the mobile terminal of users, 4G mobile communication wireless side eNodeB was placed in the sub-network. Compared with WCDMA and TD-SCDMA and other 3G wireless technology, eNodeB made the original RNC and NodeB become one. It greatly save the fixed investments and simplified the construction and maintenance and data configuration.

According to the results of site survey and network planning, to set outdoor node in the airport terminal, office area, parking lot, control tower and other areas. It adopts the way of RRU bottom allied outdoor directional antenna to achieve outdoor cover.

Because the transmitted power of outdoor directional antenna can not be too big. In order to avoid interfering normal operation of each wireless communication system of the airport, some parts of the indoor area will appear the situation of poor signal or failing to register online. In order to make up the network effectively and eliminate the signal blind area of elevator shaft, underground parking, etc, this system connects omnidirectional antenna and indoor distributed antenna under the eNodeB so as to achieve global seamless coverage of the airport.

Because the adopted equipment of this system is the same as the current network equipment of China mobile. In order to avoid appearing the problems of spectrum coverage and cross-boundary coverage with commercial $4 \mathrm{G}$ network in the process of using. It sets up the method which is different from the commercial network frequency point for each base station to distinguish from mobile commercial $4 \mathrm{G}$ network. It eliminates the problem of "robbing the network" from the source.

\section{3 user access}

The establishment of the backbone network and wireless access side network provide a good physical basis for the user access. To reduce the system cost as far as possible and not to bring extra burden to the user, system adopts the way of calling the existing SIM card ICCID code of the users directly for user registration. The administrator writes the user's SIM card information into the HLR(home location register)register in PS domain of LTE equioment to realize that users don't change card and use a mobile terminal which supports TD LTE format of China Mobile to get the result of switching freely between the commercial network and this intelligent network. Because the intelligent network connects with the commercial network by using physical gateway, the users can automatic roam between the two networks and no need to bear the risks of drop words and can not find the network, etc. 


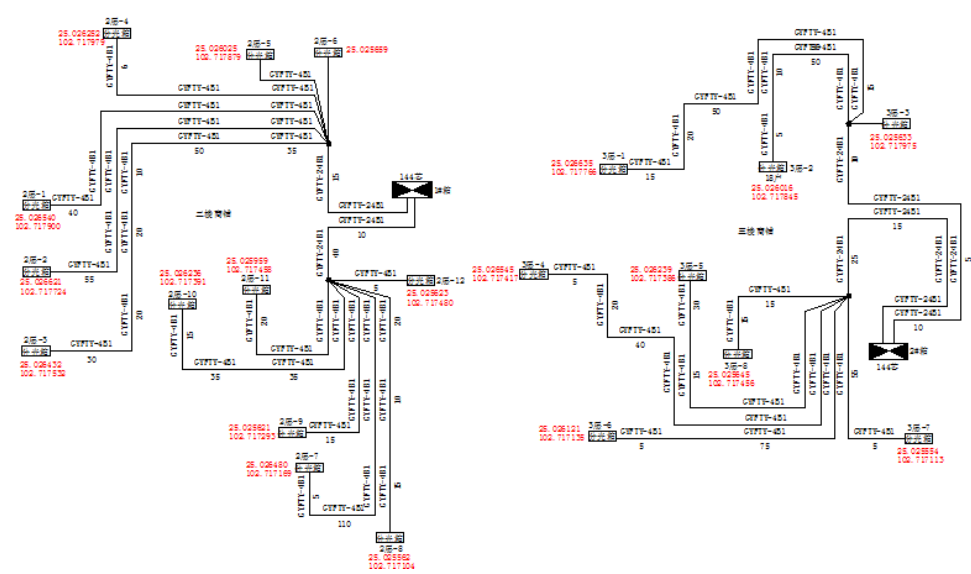

Figure 1 the overall schematic diagram of optical cable of a southwest airport

\section{3. wireless access network power control of airport intelligent information network}

Because the system is constructed in civil aviation airport area and wireless communications are highly intensive in this area, and VHF antennas, primary radar, secondary radar and other radio apparatus are numerous and commercial $4 \mathrm{G}$ networks of the operators also present to be close-set situation, multi-mobile station, multi-channel communication will cause the problems of same frequency, self-interference and near-far effect, etc[2]. There are many large buildings in the airport. The cases of multi-path fading and shadow effect caused by complex terrain and communication environment are also rather serious, and there has been the contradiction between the limited frequency resource and users need to be solved. So in order to not only avoid interfering the normal communication of existing radio, but also to realize the primary network coverage and registration target of the system design. It is particularly important to complete the wireless side power control of this system.

The power control of TD-LTE system is divided into forward power control and reverse power control, these two parts. The controlled object of the former is the transmission power of base station and mobile station plays a supplementary role. The controlled object of the latter is the transmission power of the mobile station and the base station plays a supplementary role. The categories can be divided into the open loop power control, closed loop power control and outer loop power control. In this system, two kinds of power control technology are both used.

\section{1 reverse power control}

The purpose of open loop control in reverse power control is to eliminate the free space propagation loss and shadow fading (forward and reverse are the same). It is simple and direct and it does not need to exchange the control information between mobile station and base station. It is a kind of low speed and rough control. But in the real project, because the terminal initial transmission power $(\mathrm{DBM})=-$ terminal receiving power $(\mathrm{DBM})-$ offset power + nominal transmission power offset (NOM_PWR) + access initial power offset (INIT_PWR) + interference correction factor, there may exist the same path loss for the forward and the reverse and it cannot reflect the asymmetric path loss. Because the initial judgment is based on the received total signal power, the power of mobile station received from other base station lead to the proble that the judgment is not accurate, etc[3].

Because there is unsolved and independent with forward link loss in the open loop power control. In order to realize accurate power control, it also needs the reverse closed loop power control. In this mechanism, the base station tests the signal to noise ratio $\mathrm{Eb} / \mathrm{Nt}$ from mobile phones. Comparing with threshold value $\mathrm{Eb} / \mathrm{N} 0$, it generates the power control bits to the mobile station. 0 means raising the power, 1 means reducing the power. Test instruction launches 800 times per second (i.e., updating once every $1.25 \mathrm{~ms}$ ), each instruction requests the mobile station to increase or reduce a step value of the transmission power.

It is found in practice that if the threshold value $\mathrm{Eb} / \mathrm{No}$ in the reverse closed loop power control 
does not have dynamic adjustment function, it can more effectively combine the frame error rate which directly influences the quality of speech frame error rate with the signal to noise ratio of the reverse closed loop power control. Through the change of the quality to realize real-time adjustment of the threshold value, therefore, the system also adopts the method of reverse loop power control. The reverse outer loop adjustment speed: $20 \mathrm{~ms}$, namely sampling at a rate of 50 frames per second.

\subsection{Forward Power Control}

Forward power control is mainly the forward traffic channel power which controls the sector to distribute for the mobile station with the help of the feedback information of the mobile station. On this account to reduce the forward transmission total power of this sector and to avoid the unable access of new users caused by the shortage of the forward power or appearance of the situation of unable to switch to this sector. Forward overhead channel (including the pilot frequency, synchronization, paging) is without power control [4].

\subsection{1 power setting of overhead channel}

Pilot: 15 20\% (-7.5dB:17.78\%) ; Sync: 1 2\% (-17.5dB:1.778\%) ; Paging: 10 15\% (-9dB:12.58\%)

All the powers of the forward channel are calibrated with pilot channel power. Usually to set the percentage of overhead channel to total power as Pilot: $15 \sim 20 \%(-7.5 \mathrm{~dB}: 17.78 \%)$; Sync: $1 \sim 2 \%$

(-17.5dB:1.778\%) ; Paging: 10 15\% (-9dB:12.58\%)

In order to make the covering distances of three overhead channels consistent, it should ensure that each overhead channel power is set as the following ratio:

pilot channel to residential design power: $\mathrm{XdB}$. synchronous channel to residential design power: $(\mathrm{X}-10) \mathrm{dB}$. 9.6kpbs paging channel to residential design power: $(\mathrm{X}-1.5) \mathrm{dB}$. 4.8kpbs paging channel to residential design power: $(\mathrm{X}-4.5) \mathrm{dB}$

\subsection{2 forward overload protection}

With the constant establishment of forward channel, the total power of forward transmit will keep rising. When the total power of forward transmit exceeds the rated power, it will lead to overpower work of power amplifier and a long time of overpower work will cause equipment failure and result in paralysis. In order to protect the hardware equipment, it should be in accordance with the equipment manufacturers operating manuals to set forward overload protection.

In the forward power control process which is on condition of RC3 $\sim \mathrm{RC} 5$, the mobile station every PCG $(1.25 \mathrm{~ms})$ measures the $\mathrm{Eb} / \mathrm{Nt}$ (fast power control)which is received from the monitored $\mathrm{F}-\mathrm{FCH}, \mathrm{F}-\mathrm{SCH}$. Comparing Eb/Nt value got from the measurement and $(\mathrm{Eb} / \mathrm{N} 0)$ calibration value got from forward outer loop power control algorithm to make the power control decision. Then through the reverse power control component channel of reverse pilot channel to transmit power control bits to the base station. Base station gets the power control bits. According to the corresponding power control step size and the bounds of channel power, the interior of CSM6700 chip will automatically realize the forward transmission power adjustment of each PCG. The outer loop control is completely completed by the mobile station alone. When in assignment of F-FCH or F-SCH, base station just need to assign the messages of the related parameters of outer ring algorithm in the expansion channel of the paging channel or send to the mobile station in expansion supplement channel assignment message. Mobile station is monitoring the forward basic channel $\mathrm{FCH}$. After the mobile station decrypts every $20 \mathrm{~ms}$ frame, it judges the frame whether it is good or bad. By adjusting the (Eb/N0) to achieve the calibration frame error rate FPC_FCH_FER on the forward basic channel with the condition of corresponding data rate.

Traffic channel power value algorithm as follows[5], A traffic channel power $=$ residential design power x (10 E ((traffic channel gain - 255) / 40) X2) mw.

In the practical engineering case, the initial transmission power of voice business RC3RC4RC5 forward basic channel is 179 , nominal power 2 of voice business is 175 , variation range of voice business power is 2 to 40 .

Initial transmission power of voice business is 179 . When in the soft switch of two sides, the maximum power value of each forward basic traffic channel is $175+40 / 2=195$ and the minimum power value of each forward basic traffic channel is $175-40 / 2=155$. 
Through configuration, the powers of all types of this system control to work together to make the power consumption of mobile station reduce to the minimum and increase the overall capacity of the system. It reduces the interference to the other radio communication systems and achieves the original intention of design and requirements of the system.

\section{Conclusion}

This paper is according to the demand of the airport intelligent information network of civil aviation. It analyzes the technical parameters and requirements of backbone network and wireless side mobile network if the system and establishes the principle of using the way of "optical network + TD-LTE" to set up the communication network and this paper introduces overall process of construction of core network and wireless side of the system. In this paper, there have analyzed the necessity and specific measures of doing power control to system wireless side Through case analysis, the methods of data collection and statistics and scientific computing to analyze and evaluate the two kinds of power control strategies of forward power control and reverse power control and configure and verify in specific equipment in accordance with the relevant technical manuals. To establish the known optimization solution creatively and realize effective power control of wireless side network of airport intelligent information network. It provides beneficial reference and theoretical support for engineering practice .

\section{References:}

[1] Xu Xiukai:Aviation Communication and International Flight(Sichuan Science and Technology Publishing House China 2012)

[2] He Lei:Civil Aviation Airport Ground Service(Chemical Industry Publishing House China 2014)

[3]Yuan Yiping:LTE/LTE-Advanced Key Technology and System Performance(People's Posts and Telecommunications Publishing House China 2014)

[4]Zhang Shouguo:LTE Wireless Network Optimization Practice(People's Posts and Telecommunications Publishing House China 2014)

[5]Hendrik Berndt:Mobile Service Technology based on the 4G System( China Machine Press China 2013) 